\title{
Entanglement of scales as a possible mechanism for decoherence and thermalization in relativistic heavy ion collisions
}

\author{
S.V. Akkelin ${ }^{a}$, Yu.M. Sinyukov ${ }^{a, b}$ \\ (a) Bogolyubov Institute for Theoretical Physics, \\ Metrolohichna str. 14b, 03680 Kiev, Ukraine \\ (b) ExtreMe Matter Institute EMMI, \\ GSI Helmholtz Zentrum für Schwerionenforschung, D-64291 Darmstadt, Germany
}

\begin{abstract}
Despite the fact that a system created in relativistic heavy ion collisions is an isolated quantum system, which cannot increase its entropy in the course of unitary quantum evolution, hydrodynamical analysis of experimental data seems to indicate that the matter formed in the collisions is thermalized very quickly. Based on common consideration of hydrodynamics as an effective theory in the domain of slow- and long-length modes, we discuss the physical mechanisms responsible for the decoherence and emergence of the hydrodynamic behavior in such collisions, and demonstrate how such physical mechanisms work in the case of the scalar field model. We obtain the evolution equation for the Wigner function of a long-wavelength subsystem that describes its decoherence, isotropization, and approach to thermal equilibrium induced by interaction with short-wavelength modes. Our analysis supports the idea that decoherence, quantum-to-classical transition and thermalization in isolated quantum systems are attributed to the experimental context, and are related to a particular procedure of decomposition of the whole quantum system into relevant and irrelevant from an observational viewpoint subsystems.
\end{abstract}

PACS numbers: 25.75.-q, 03.65.Yz 


\section{INTRODUCTION}

The unique and very ambitious program on the creation and study of a small part of the early universe in laboratories is being carried out at the Relativistic Heavy Ion Collider (RHIC) and the Large Hadron Collider (LHC). In collisions between nuclei at these machines a huge number of created particles forms a rapidly expanding quark-gluon and/or hadron systems within space-time scales $10^{-14} \mathrm{~m}$ and $10^{-22} \mathrm{~s}$. One of the most important results obtained in these experiments is that hydrodynamic models with nearly perfect fluid describe well the observables in relativistic heavy ion collisions. The best agreement with data is achieved if very early thermalization times $(\lesssim 1 \mathrm{fm}$ ) of the produced quark-gluon matter are assumed [1].

In spite of a significant recent progress in the study of early time dynamics of quarkgluon matter produced in high energy heavy ion collisions, thermalization of the matter created in $A+A$ collisions remains the great and still unresolved mystery (see, e.g., Ref. [2]). It seems that, at least partially, this is so because a system created in each nucleusnucleus collision can be considered as an isolated quantum system that does not interact with external environment, and unitary quantum evolution of an isolated system cannot increase its entropy, no matter what happens during this evolution (e.g., deconfinement, etc.), and no matter how large and complex the system is.

To avoid the conceptual problems with thermalization of an isolated quantum system, some approaches utilize classical approximation for early time dynamics in heavy ion collisions. We mention here the approaches that are based on classical picture of on-mass-shell particles: Boltzmann gas of particles with short-range interactions (see, e.g., Ref. [3] ), and plasma particles with long-range interactions (see, e.g., Ref. [4]). The more sophisticated approach for a description of early time dynamics and equilibration processes in $A+A$ collisions utilizes the initial state, which follows from the color glass condensate (CGC) effective field theory (for recent reviews see, e.g., Ref. [5] and references therein). This describes degrees of freedom in the colliding nuclei as highly occupied gluon fields with small gauge coupling that are produced by the statistical ensemble of classical color sources on an event-by-event basis. Such an initial state corresponds to the Glauber coherent state [6] that minimizes the uncertainty relation. Then, because for such a state the classical $\hbar \rightarrow 0$ limit (see, e.g., Ref. [7]) is equivalent to the limit when the coupling constant tends to zero 
and the field's momentum and coordinate expectation values tend to infinity, one utilizes classical Yang-Mills equations with fluctuating initial conditions as suitable approximation for the description of early time dynamics in relativistic heavy ion collisions (see, e.g., Ref. [8] and references therein).

It is still unclear whether or not such an approach can result in proper early thermalization in $A+A$ collisions. Moreover, even if the approach, based on the classical picture, will result in early thermalization, it does not help to understand this phenomenon in $A+A$ collisions from the first principles. This is so because a system created in an $A+A$ collision is inherently quantum 1 , and its initial state is, in fact, quantum superposition of the Glauber coherent states. Only if the different Glauber coherent states could be distinguished experimentally as separate initial states of colliding nuclei, the initial condition can be substituted by the corresponding statistical mixture. If this is not the case, then such a substitution is unjustified. One more shortcoming with such an approach is that unlike a classical system, where chaotic behavior can appear due to extreme sensitivity of a complex system to the initial conditions, in a quantum system the unitarity of the Schrödinger evolution preserves all scalar products and, so, all the "distances" between quantum state vectors during the time, and no chaotic behavior is possible. Therefore, even for a quasiclassical initial condition the quasiclassical approximation can be destroyed relatively quickly for systems that exhibit classical dynamical (deterministic) chaos. Indeed, it was demonstrated that this happens after a time that is only logarithmic in the Plank constant [10] resulting in a noticeable deviation between classical and quantum expectation values [11]. Then, loosely speaking, the chaos seen in the approximate classical dynamics of isolated quantum system is an artifact of the approximations. Only decoherence caused by the environment can substantially reduce the discrepancy between quantum and classical expectation values and restore the quantumclassical correspondence for a classically chaotic system [10-12].

Perhaps, a hope that the anti-de-Sitter/conformal-field-theory (AdS/CFT) correspondence [13] (for reviews see Refs. [14, 15]) can help to understand thermalization in a closed quantum system is one of the reasons why this approach, which attempts to explain the origin of decoherence and thermalization in $A+A$ collisions, has recently attracted much attention in the heavy ion community. While AdS/CFT correspondence does not take place

${ }^{1}$ For any fixed $\hbar$, even if the classical and quantum expectation values coincide at the moment, they will diverge from each other after some time, except for the specific case of the Gaussian interaction (see, e.g., Ref. [9]). 
for QCD, it is generally believed that it provides correct qualitative picture of QCD dynamics in the strongly coupled regime 2 The AdS/CFT correspondence is based on the holographic gauge/string duality between four-dimensional (4D) quantum field gauge theory such as $N=4$ super Yang-Mills gauge theory (which is a conformal field theory), and five-dimensional quantum string theory. Therefore, the AdS/CFT correspondence is also sometimes called a gauge/string duality. The duality means an exact equivalence between two theories, i.e., it means that any calculated quantity can be expressed in terms of a dual partner theory. In practice, however, calculations in dual 5D quantum string theory are possible only under some limitations, which from the QCD viewpoint means that $N_{c} \rightarrow \infty$ and $\lambda \rightarrow \infty$, where $N_{c}$ is the number of colors and $\lambda$ is the QCD coupling constant. Under such conditions a gauge/string duality is reduced to a gauge/gravity duality between 4D quantum gauge theory and 5D classical gravity theory. In this correspondence, the radial coordinate $r$ of additional spatial direction can be associated with the renormalization group energy scale (energy cutoff scale) in the gauge field theory [17], and asymptotically high values of radius parameter correspond to gauge field theory with asymptotically high energy cutoff. Therefore, the AdS/CFT correspondence can be treated as geometrization of a renormalization group.

The phenomenon of thermalization of 4D quantum field theory in this approach is then associated with the irreversible process of black hole (and corresponding event horizon with non-zero entropy) formation [15]. Specifically, it was found that the long-wavelength (smoothed over short-scales) approximation of metrics induced by a large stationary black hole corresponds to a thermal state of the gauge quantum field theory, and the longwavelength approximation of metrics induced by a large nearly stationary black hole corresponds to a nearly perfect hydrodynamical structure of the expectation value of the energy momentum tensor of gauge quantum fields. The latter duality is sometimes named as the fluid/gravity correspondence [18]. Such a fluid/gravity correspondence is a useful tool to calculate viscosity for strongly interacting locally equilibrated systems [15]. But the question appears: does such duality explain the thermalization process in $A+A$ collisions?

First, note that sometimes, to support idea of "black hole thermalization", one appeals to the well known and well understood Unruh effect [19] (for review see, e.g., Ref. [20]). It is

${ }^{2}$ Note here, that the applicability of such a regime for the early stage dynamics in relativistic heavy ion collisions is questionable, see, e.g., Ref. [16]. 
noteworthy that the dual description of this effect from the point of view of an accelerating observer and inertial observer gives the same result: thermalization of an uniformly accelerated (in inertial reference system) particle detector [20, 21] due to quantum interactions. However, one needs to remember that conditions, for which the Unruh effect takes place, mean that the accelerated detector is an open system that is accelerated by some external forces, and such a thermalization cannot be observed for a closed isolated system with an accelerated subsystem - detector [22]. This point was missed in recent attempts to explain thermalization in $A+A$ collisions by means of the Unruh mechanism [23].

Second, note that AdS/CFT correspondence cannot be treated as the origin of thermalization and entropy creation in dual 4D quantum field theory because the latter is an ordinary quantum field theory in flat space-time, and so cannot produce entropy in the course of reversible and unitary quantum evolution. Then, based on general principles of quantum theory one can infer that the fluid/gravity correspondence is valid for some decomposition of the whole quantum system into separate subsystems: it is well known that while entropy of the whole isolated quantum system remains constant under the time evolution, entanglement entropies of its subsystems can increase. Indeed, recent studies in time-dependent AdS/CFT based on a holographic formula of the entanglement entropy [24] demonstrate that black-hole formation in AdS dual can be associated with an increase of the entanglement entropy in CFT dual [25] (for recent reviews see Ref. [26]), but the latter takes place only after splitting of CFT dual into spatially separated subsystems with a quantum quench of one of them at a specific instant of time.

In what follows, we adopt the standpoint that (entanglement) entropy production in an isolated quantum system can take place only after its decomposition into subsystems, and that the specific way of separation of the closed system into subsystems depends on a certain experimental context (i.e., it is related with "relevant" observables). Instead of decomposition of the system into separated-in-space subsystems, we split the system into longwavelength modes and short-wavelength modes subsystems, and treat the long-wavelength modes subsystem as the relevant one and the short-wavelength modes subsystem as the environment. Such a decomposition seems to be appropriate for the experimental context in $A+A$ collisions, and is in agreement with common consideration of hydrodynamics as the effective theory in the domain of slow- and long-length modes (see, e.g., Ref. [27]). Inasmuch as the aim of this paper is partly methodological, we will focus mainly on the general 
quantitative features of the evolution of long wavelength quantities in a simple scalar field theory model to investigate the physical mechanisms responsible for the decoherence and emergence of the hydrodynamic behavior in $A+A$ collisions.

\section{DECOHERENCE AND APPROACH TO EQUILIBRIUM OF THE LONG WAVELENGTH OBSERVABLES}

Let us start with pointing out that necessary condition for emergence of hydrodynamic behavior is the decoherence, i.e., suppression of interference of some set of variables that allows one to use local densities to describe a system's dynamics. It is well known that an open system can be decohered (i.e., its state can be approximately diagonalized in some basis) and can acquire classical properties due to interactions with its environment containing the many degrees of freedom that are ignored from an observational point of view (for review see [28] and references therein). Note that such a decoherence due to interactions between the system and its environment is formulated entirely quantum mechanically, and globally the quantum mechanical superposition remains unchanged, as required by the unitarity of the evolution of the total wave function. In contrast with such an environment-induced decoherence, in a relativistic nucleus-nucleus collision the system remains isolated after preparation and until an observation at a large time $t_{\text {out }}$ is performed. However, it is well known that while decoherence of an isolated system is impossible, the decoherence of its subsystems is still possible: while the state of the whole system remains pure, the state of a subsystem of a composite system can be described as improper mixture [29] represented by the partial trace of the statistical operator of the composite system in a pure state (proper mixture means incomplete knowledge for a pure state, and, typically, represents a statistical ensemble). The key point here is quantum entanglement: interacting quantum subsystems become entangled in the course of unitary evolution of the system as a whole and, as a result, the quantum states of subsystems become mixed states. It is noteworthy that such mixed states generation has nothing to do with the formation of statistical ensembles when the weights of the states have no relation to the exact dynamical equations.

Note that because of the quantum non-separability [29] a closed system can be resolved into parts ("subsystems") in various ways. Different splittings result in complementary descriptions of a system, and the state of a whole quantum system can not be inferred 
from the states of its parts unlike the state of a composite classical system, which can always be reconstructed from the known states of its parts. Decoherence and, perhaps, thermalization thus arise from the description of the system by an observer who at the selected measurements and data analysis has access only to subsystem degrees of freedom, while residual degrees of freedom are entangled with the subsystem but remain unobserved. The state of the whole system, however, remains pure, and its entropy remains zero: due to the quantum entanglement, the entropy of a whole quantum system is not equal to the sum of the entropies of its parts that are defined as the von Neumann entropies of the corresponding reduced density matrices. In this kind of process, the equilibrium state of the relevant subsystem is just a state when its entropy reaches a maximum due to the build-up of entanglement of the considered system with its environment induced by the interactions [30].

Then, to explain the success of hydrodynamics in $A+A$ collisions, one can assume that a system created in a relativistic $A+A$ collision can be decomposed into the fast short-length modes that represent irrelevant (i.e., observationally inaccessible or ignored) degrees of freedom, and slow long-length modes that represent relevant (i.e., observationally accessible) degrees of freedom. The former act as environment and can ensure decoherence and approach to (local) equilibrium for the latter [31] (see also Ref. [32]). Such a splitting is conditioned by the experimental context because of limited region and accuracy in a measurement of relevant observables (e.g., particle momentum spectra) and, also, because not all possible observables are measured (e.g., not all $N$-particle correlations, quantum interference effects, etc.).

The evolution of the relevant subsystem of closed system is studied usually by means of utilizing powerful mathematical methods, e.g., by means of the projection operator technique (for review see, e.g., Ref. [33]). Note, however, that application of these methods to nonequilibrium quantum field theory is usually rather complicated and sometimes ambiguous, and physics is often hidden by mathematical formalisms. Therefore, for illustrative purposes, we will utilize here a more heuristic coarse graining approach aiming to make clear origin of decoherence of relevant observables in $A+A$ collisions and their subsequent evolution towards equilibrium.

Due to the complexity of the problem, we restrict ourselves to a $\varphi^{4}$ quantum field model, 
whose dynamics is determined by the Lagrangian density

$$
L=\frac{1}{2} \partial_{\mu} \varphi \partial^{\mu} \varphi-\frac{\lambda}{4 !} \varphi^{4},
$$

where $\lambda$ is coupling constant. In the following, we utilize the Heisenberg representation. Expectation values are defined as $\langle O\rangle=S p(\hat{\rho} O)$, where $\hat{\rho}$ denotes the statistical operator associated with an initial (pure) state of the system.

The expectation value of the energy momentum tensor, $\left\langle T_{\mu \nu}\right\rangle$, satisfies to conservation equations

$$
\partial^{\mu}\left\langle T_{\mu \nu}\right\rangle=0
$$

which follows from the field evolution equation. Many studies of the $\left\langle T_{\mu \nu}\right\rangle$ evolution were based on classical field approximation of the energy momentum tensor,

$$
\left\langle T^{\mu \nu}(x)\right\rangle \approx T^{\mu \nu}[\langle\varphi\rangle]=\partial^{\mu}\langle\varphi\rangle \partial^{\nu}\langle\varphi\rangle-g^{\mu \nu}\left[\frac{1}{2}\left(\partial_{\alpha}\langle\varphi\rangle\right)^{2}-\frac{\lambda}{4 !}\langle\varphi\rangle^{4}\right] .
$$

It is worth to note here that such an approximation does not mean that the evolution of $\left\langle T_{\mu \nu}\right\rangle$ proceeds as in classical field theory. This is so because the expectation value of the field, $\langle\varphi\rangle$, is governed by the equation

$$
\partial^{\mu} \partial_{\mu}\langle\varphi\rangle=-\frac{\lambda}{3 !}\left\langle\varphi^{3}\right\rangle
$$

where $\left\langle\varphi^{3}\right\rangle$ in the right hand side contains correlations of quantum fluctuations. Classical evolution for $\left\langle T_{\mu \nu}\right\rangle$ can be obtained if $\left\langle\varphi^{3}\right\rangle$ is approximated by $\langle\varphi\rangle^{3}$, which leads to the reversible classical evolution equation for $\langle\varphi\rangle$. It is well known, however, that classical equations approximate the underlying microscopic quantum dynamics for the very special initial coherent (Glauber) state [6] and during a limited time period only. On the other hand, if instead of the whole system we consider the relevant subsystem, and associate the latter with long wavelength modes (i.e., with momentum scales $k$ smaller than the some characteristic scale $k^{\star}$ ), then utilization of the classical approximation for expectation values of long wavelength observables can be justified, and quantum fluctuations can be accounted for short-length modes only 3

\footnotetext{
3 Note that quantum correlations are suppressed for the long-wavelength modes because long-wavelength mode operators are, in fact, smeared operators, and the canonical commutation relation for the smeared conjugated operators tends to zero if the scale of averaging tends to infinity (see, e.g. Ref. [34] and references therein). This allows one to use classical approximation the long-wavelength modes evolution after decoherence, the latter is necessary but not sufficient condition for classical approximations.
} 
Let us split the quantum field $\varphi$ at $t=t_{0}$ into long-wavelength modes $\varphi_{L}^{t_{0}}$, and shortwavelength modes $\varphi_{S}^{t_{0}}: \varphi=\varphi_{L}^{t_{0}}+\varphi_{S}^{t_{0}}$. We assume that the initial long wavelength field, $\varphi_{L}^{t_{0}}$, corresponds to a convolution of field operator $\varphi$ with a "window" function $W_{V}, \int W_{V}=1$, which makes smoothing/averaging of the field over a domain of size $V=1 / k^{* 3}$,

$$
\varphi_{L}^{t_{0}}\left(x, t_{0}\right)=\int d^{3} x^{\prime} W_{V}\left(x-x^{\prime}\right) \varphi\left(x^{\prime}, t_{0}\right)
$$

Also, we split a state of the system into $L$ and $S$ subsystems: $\hat{\rho}_{L} \otimes \hat{\rho}_{S}$, assuming that observables correspond to operators acting on $L$ states only.

The evolution equation for expectation value of long-length modes with initial condition defined according to (5) reads

$$
\partial^{\mu} \partial_{\mu}\left\langle\varphi_{L}^{t_{0}}\right\rangle=-\partial^{\mu} \partial_{\mu}\left\langle\varphi_{S}^{t_{0}}\right\rangle-\frac{\lambda}{3 !}\left\langle\left(\varphi_{L}^{t_{0}}+\varphi_{S}^{t_{0}}\right)^{3}\right\rangle
$$

One can see that in the course of evolution the initially smeared field becomes dependent on short-wavelength modes. This is a manifestation of quantum entanglement in the Heisenberg picture. To follow the evolution of the corresponding observables, one needs to make repeated in time splitting of the whole quantum system into the corresponding subsystems, in the Heisenberg picture this means that one needs to make repeated redefinition of the corresponding observables (this is reminiscent of the familiar repeated randomness assumption in the Boltzmann kinetics).

Then, to calculate observables associated with long wavelength modes, one needs to supplement this exact motion with an operation that prevents the state to deviate too much from $L$. This can be done by dividing the evolution into time intervals, and choosing initial conditions for each time step with $\varphi_{L}^{t_{i}}$ being replaced at the time $t_{i+1}=t_{i}+\delta t$ by the associated $\varphi_{L}^{t_{i+1}}\left(x, t_{i+1}\right)=\int d^{3} x^{\prime} W_{V}\left(x-x^{\prime}\right)\left(\varphi_{L}^{t_{i}}\left(x^{\prime}, t_{i+1}\right)+\varphi_{S}^{t_{i}}\left(x^{\prime}, t_{i+1}\right)\right)$. Then for $t_{i+1}<t<t_{i+2}=t_{i+1}+\delta t$

$$
\partial^{\mu} \partial_{\mu}\left\langle\varphi_{L}^{t_{i+1}}\right\rangle=-\partial^{\mu} \partial_{\mu}\left\langle\varphi_{S}^{t_{i+1}}\right\rangle-\frac{\lambda}{3 !}\left\langle\left(\varphi_{L}^{t_{i+1}}+\varphi_{S}^{t_{i+1}}\right)^{3}\right\rangle
$$

and we have piecewise continuous description of $L$-modes evolution 4 Now, let us neglect in each $\delta t$-interval contribution of long-scale quantum fluctuations and contribution of the

\footnotetext{
${ }^{4}$ Note that an exact equation of motion for the relevant variables may be obtained by this procedure if their characteristic time scale are much larger than the time scales associated with the irrelevant variables and if the time $\delta t$ is chosen in between $[35]$.
} 
short wavelength modes into the right-hand side of the evolution equations. Then we get the chain of equations

$$
\begin{array}{r}
\partial^{\mu} \partial_{\mu}\left\langle\varphi_{L}^{t_{0}}\right\rangle=-\frac{\lambda}{3 !}\left\langle\varphi_{L}^{t_{0}}\right\rangle^{3}, t_{0}<t<t_{1}, \\
\cdots \\
\partial^{\mu} \partial_{\mu}\left\langle\varphi_{L}^{t_{n}}\right\rangle=-\frac{\lambda}{3 !}\left\langle\varphi_{L}^{t_{n}}\right\rangle^{3}, t_{n-1}<t<t_{n},
\end{array}
$$

which approximates piecewise continuous description of $L$-modes till some time $t_{n}$. Note that the projection times set, $\left\{t_{i}\right\}$, is not uniquely defined and can vary in some intervals allowed by dynamics. Therefore, such a piecewise continuous description means that we have, in fact, a set of different histories of the $L$-modes evolution with randomly chosen projection times and, so, random expectation values of $L$-modes. Such a set of piecewise continuous evolutions can be approximated by the continuous one,

$$
\partial^{\mu} \partial_{\mu}\left\langle\varphi_{L}\right\rangle_{\xi}=-\frac{\lambda}{3 !}\left(\left\langle\varphi_{L}\right\rangle_{\xi}^{3}+\xi\right),
$$

where $\xi$ accounts for random discontinuity $\left\langle\varphi_{L}^{t_{i}}\right\rangle\left(t_{i+1}\right) \neq\left\langle\varphi_{L}^{t_{i+1}}\right\rangle\left(t_{i+1}\right)$ and, so, is associated with fluctuations of the expectation value of long wavelength modes. As we discussed above, such a discontinuity is caused by the interaction of long wavelength modes with the short wavelength ones, in particular, by the interaction with the short-scale quantum fluctuations that typically are more enhanced than the long-scale ones. Because the information transferred towards the irrelevant variables is discarded at the beginning of each time interval, $\xi$ becomes a stochastic "noise" variable, and induces a continuous time random walk stochastic dynamics for $\left\langle\varphi_{L}\right\rangle_{\xi}$. Then, to get true long wavelength observables without "trembles" that are associated with different projection histories, one needs to average such observables over $\xi$. Such an averaging means, in fact, smearing over the time interval $\delta t$ for set of projection histories, and is not associated with statistical ensemble of initial events. The necessary condition for hydrodynamical approximation to be valid is the allowance to neglect, after such an averaging, non-conservation of energy momentum due to interactions with short wavelength modes, i.e., to make the assumption that such an interaction results mostly in the information loss.

Direct calculation of $T_{\mu \nu}\left[\left\langle\varphi_{L}\right\rangle_{\xi}\right]$ based on evolution equations for $\left\langle\varphi_{L}\right\rangle_{\xi}$ is a rather uneasy task, which can hardly be done analytically. Therefore, here we proceed in a more heuristic way and express $T_{\mu \nu}\left[\left\langle\varphi_{L}\right\rangle_{\xi}\right]$ through the expectation value of the corresponding Wigner 
operator (see, e.g., Ref. [36]), and obtain for the latter a kinetic transport equation. Let us define the (reduced) Wigner function describing the state of the long-wavelength modes, $N_{L}(x, p)$, as

$$
N_{L}(x, p)=\sum_{\xi} N_{L}^{\xi}(x, p)
$$

where

$$
N_{L}^{\xi}(x, p)=(2 \pi)^{-4} \int d^{4} v e^{-i p v}\left\langle\varphi_{L}\right\rangle_{\xi}\left(x+\frac{1}{2} v\right)\left\langle\varphi_{L}\right\rangle_{\xi}\left(x-\frac{1}{2} v\right),
$$

and symbol $\sum_{\xi}$ means that we perform in (11) the average with respect to random $\xi$ fluctuations, as was discussed above.

Then the energy momentum tensor of long wavelength modes, $T_{\mu \nu}^{L}(x)$, can be defined as averaged over $\xi$ classical approximation of $\left\langle T_{\mu \nu}\right\rangle_{L}$ :

$$
\left\langle T_{\mu \nu}\right\rangle_{L} \approx T_{\mu \nu}^{L}(x)=\sum_{\xi} T_{\mu \nu}\left[\left\langle\varphi_{L}\right\rangle_{\xi}\right]
$$

where $T_{\mu \nu}\left[\left\langle\varphi_{L}\right\rangle_{\xi}\right]$ is written as in (3) but with substitution $\langle\varphi\rangle \rightarrow\left\langle\varphi_{L}\right\rangle_{\xi}$. By means of the Wigner function (12) one can rewrite $T_{\mu \nu}\left[\left\langle\varphi_{L}\right\rangle_{\xi}\right]$ as [36]

$$
\begin{aligned}
T_{\mu \nu}\left[\left\langle\varphi_{L}\right\rangle_{\xi}\right]= & \int \mathrm{d}^{4} p\left(p_{\mu} p_{\nu}+\frac{1}{4} \partial_{x^{\mu}} \partial_{x^{\nu}}-\frac{1}{2} g_{\mu \nu}\left(p^{2}+\frac{1}{4} \partial_{x}^{2}\right)\right) N_{L}^{\xi}(x, p) \\
& +\frac{\lambda g_{\mu \nu}}{4 !} \int \mathrm{d}^{4} p \mathrm{~d}^{4} p^{\prime} N_{L}^{\xi}(x, p) N_{L}^{\xi}\left(x, p^{\prime}\right) .
\end{aligned}
$$

Using (10), we obtain the following time evolution of the Wigner function:

$$
\begin{array}{r}
p_{\mu} \partial^{\mu} N_{L}(x, p)= \\
2(2 \pi)^{4} \\
\sum_{\xi} \int d^{4} v e^{-i p v}\left(\rho_{\xi}\left(x-\frac{v}{2}\right)\left\langle\varphi_{L}\right\rangle_{\xi}\left(x+\frac{v}{2}\right)-\left\langle\varphi_{L}\right\rangle_{\xi}\left(x-\frac{v}{2}\right) \rho_{\xi}\left(x+\frac{v}{2}\right)\right) .
\end{array}
$$

Here

$$
\rho_{\xi}=-\frac{\lambda}{3 !}\left(\left\langle\varphi_{L}\right\rangle_{\xi}^{3}+\xi\right)
$$

Aiming to derive the kinetic equation for the Wigner function, let us rewrite the above equation in the form

$$
\begin{aligned}
& p_{\mu} \partial^{\mu} N_{L}(x, p)= \\
& \frac{i}{2(2 \pi)^{4}} \sum_{\xi} \int d^{4} v e^{-i p v}\left\langle\varphi_{L}\right\rangle_{\xi}\left(x+\frac{v}{2}\right)\left\langle\varphi_{L}\right\rangle_{\xi}\left(x-\frac{v}{2}\right)\left(\varrho_{\xi}\left(x-\frac{v}{2}\right)-\varrho_{\xi}\left(x+\frac{v}{2}\right)\right) \text {, }
\end{aligned}
$$


where

$$
\varrho_{\xi}=\frac{\rho_{\xi}}{\left\langle\varphi_{L}\right\rangle_{\xi}}
$$

Then, performing the Tailor expansion of $\left(\varrho_{\xi}\left(x-\frac{v}{2}\right)-\varrho_{\xi}\left(x+\frac{v}{2}\right)\right)$ in powers of $v$ and integrating over $v$, we get

$$
p_{\mu} \partial^{\mu} N_{L}(x, p)=\frac{1}{4} \sum_{\xi} \partial^{\mu} \varrho_{\xi}(x) \frac{\partial}{\partial p^{\mu}} N_{L}^{\xi}(x, p)+\sum_{\xi} \Phi_{\xi}(x, p),
$$

where we used (12) and $\sum_{\xi} \Phi_{\xi}(x, p)$ includes all high derivatives terms of the Tailor expansion. Let us make the natural assumption that averaging over $\xi$ reduces high derivatives terms and allows one to neglect the last term in Eq. (19). Then in such an approximation

$$
p_{\mu} \partial^{\mu} N_{L}(x, p)=\frac{1}{4} \sum_{\xi} \partial^{\mu} \varrho_{\xi}(x) \frac{\partial}{\partial p^{\mu}} N_{L}^{\xi}(x, p),
$$

and $N_{L}^{\xi}(x, p)$, as follows from (11), is governed by the equation

$$
p_{\mu} \partial^{\mu} N_{L}^{\xi}(x, p)=\frac{1}{4} \partial^{\mu} \varrho_{\xi}(x) \frac{\partial}{\partial p^{\mu}} N_{L}^{\xi}(x, p)
$$

Let us define

$$
\begin{gathered}
\delta N_{L}^{\xi}=N_{L}^{\xi}-N_{L}, \\
\delta \varrho_{\xi}=\varrho_{\xi}-\varrho
\end{gathered}
$$

here $\varrho=\sum_{\xi} \varrho_{\xi}$. Then, using (22) and (23), Eq. (19) reads

$$
p_{\mu} \partial^{\mu} N_{L}(x, p)=\frac{1}{4} \partial^{\mu} \varrho(x) \frac{\partial}{\partial p^{\mu}} N_{L}(x, p)+\frac{1}{4} \sum_{\xi}\left[\partial^{\mu} \delta \varrho_{\xi}(x) \frac{\partial}{\partial p^{\mu}} \delta N_{L}^{\xi}(x, p)\right] .
$$

Now one needs to calculate the second term in the right-hand side of the above equation. Subtracting (24) from (21) and keeping only the lowest terms in $\delta$, one can get

$$
p_{\mu} \partial^{\mu} \delta N_{L}^{\xi}(x, p)=\frac{1}{4} \partial^{\mu} \delta \varrho_{\xi}(x) \frac{\partial}{\partial p^{\mu}} N_{L}(x, p)+\frac{1}{4} \partial^{\mu} \varrho(x) \frac{\partial}{\partial p^{\mu}} \delta N_{L}^{\xi}(x, p),
$$

which can be rewritten as

$$
\begin{aligned}
\delta N_{L}^{\xi}(x, p)=\delta N_{L}^{(f r e e) \xi}(x, p)+ & \frac{1}{4} \int d^{4} y G_{p}(x-y) \delta \varrho_{\xi}^{\mu}(y) \frac{\partial}{\partial p^{\mu}} N_{L}(y, p)+ \\
& \frac{1}{4} \int d^{4} y G_{p}(x-y) \varrho^{\mu}(y) \frac{\partial}{\partial p^{\mu}} \delta N_{L}^{\xi}(y, p) .
\end{aligned}
$$


Here $\varrho^{\mu} \equiv \partial^{\mu} \varrho, \delta \varrho_{\xi}^{\mu} \equiv \partial^{\mu} \delta \varrho_{\xi}, \delta N_{L}^{(\text {free }) \xi}(x, p)$ is the general solution of the homogeneous equation:

$$
p_{\mu} \partial^{\mu} \delta N_{L}^{(\text {free }) \xi}(x, p)(x, p)=0
$$

and

$$
\begin{array}{r}
p^{\mu} \partial_{\mu} G_{p}(x)=\delta^{(4)}(x), \\
G_{p}(x)=p_{0}^{-1} \Theta(t) \delta^{(3)}\left(\mathbf{r}-\left(\mathbf{p} / p_{0}\right) t\right)
\end{array}
$$

Let us assume that initially $\delta N_{L}^{\xi}=0$. Then $\delta N_{L}^{(\text {free }) \xi}=0$ and one can see from Eq. (26) that in lowest order in $\delta$

$$
\delta N_{L}^{\xi}(x, p)=\frac{1}{4} \int d^{4} y G_{p}(x-y) \delta \varrho_{\xi}^{\mu}(y) \frac{\partial}{\partial p^{\mu}} N_{L}(y, p)
$$

In such an approximation (24) reads

$$
\begin{array}{r}
p_{\mu} \partial^{\mu} N_{L}(x, p)= \\
\frac{1}{4} \partial^{\mu} \varrho(x) \frac{\partial}{\partial p^{\mu}} N_{L}(x, p)+\frac{1}{16} \frac{\partial}{\partial p^{\mu}} \int d^{4} y G_{p}(x-y) \sum_{\xi}\left[\delta \varrho_{\xi}^{\mu}(x) \delta \varrho_{\xi}^{\nu}(y)\right] \frac{\partial}{\partial p^{\nu}} N_{L}(y, p) .
\end{array}
$$

In general, we cannot compute exactly the contributions of the fluctuations (otherwise we could solve exactly the model): approximations are necessary. Then, to proceed further we have to specify the stochastic properties of the random quantities $\delta \varrho_{\xi}^{\mu}(x)$. We take the simplest ansatz assuming that the backreaction is negligible

$$
\sum_{\xi}\left[\delta \varrho_{\xi}^{\mu}(x) \delta \varrho_{\xi}^{\nu}(y)\right]=\tau^{\mu \nu}(x, y) \delta\left(t_{x}-t_{y}\right)
$$

The assumption of a $\delta$-function in time difference means that the auto-correlation time of the fluctuations is small compared to the time scale of the motion of the averaged fields. The fluctuations thus appear as uncorrelated on the time scale of the motion of the averaged fields. This assumes a clear separation of scales between the short time scale of irrelevant degrees of freedom, and the long time scale which characterizes the dynamics of the relevant degrees of freedom.

Then

$$
p_{\mu} \partial^{\mu} N_{L}(x, p)=\frac{1}{4} \partial^{\mu} \varrho(x) \frac{\partial}{\partial p^{\mu}} N_{L}(x, p)+\frac{1}{16} \tau^{\mu \nu} \frac{\partial}{\partial p^{\mu}} \frac{1}{p_{0}} \frac{\partial}{\partial p^{\nu}} N_{L}(x, p)
$$


here $\tau^{\mu \nu} \equiv \tau^{\mu \nu}(x, x)$. As usual, the irreversible transport equation for relevant subsystem is valid only for finite time scales where the short-memory approximation (i.e., "white noise" approximation) is justified. It is worth to note the similarity of Eq. (33) with the Fokker-Plank equation, the latter is often utilized for a description of the approach to (local) equilibrium. In the utilized approximation, see Eqs. (9) and (10), we do not account for explicit contribution of short-wavelength modes, so $\sum_{\xi} \xi=0$ and $\varrho=\sum_{\xi} \varrho_{\xi}=-\frac{\lambda}{3 !}\left\langle\varphi_{L}\right\rangle^{2}$. Then the first term in r.h.s. of Eq. (33) is reduced to a familiar Vlasov term, and the second term in r.h.s. of Eq. (33) is associated with correlators of fluctuations induced by interactions with short-length modes. In such an approximation, the above equation cannot describe thermalization, but it still can describe process of momentum isotropization and spatiotemporal decoherence of the long-length modes, which precedes thermal equilibration acting on a shorter time scale and is a necessary condition for thermalization and hydrodynamics.

Isotropization of the relevant subsystem can happen, evidently, only because of interactions with irrelevant modes. In more mathematical terms, it can happen if the diffusion term, which is associated with correlators of fluctuations, has appropriate properties. Namely, let us assume that $\tau^{\mu \nu} \sim \delta^{\mu \nu}$, i.e., the corresponding fluctuations are isotropic. Then, to find a steady (quasistationary) state, we suppose that r.h.s. of Eq. (33) is equal to zero:

$$
\begin{gathered}
\frac{1}{2} \partial^{0} \varrho(x) \frac{\partial}{\partial p_{0}^{2}} N_{L}(x, p)+\frac{1}{4} \frac{\partial}{\partial p_{0}^{2}} \frac{\partial}{\partial p_{0}^{2}} \tau^{00} N_{L}(x, p)+ \\
\frac{1}{4 p_{0}} \partial^{i} \varrho(x) \frac{\partial}{\partial p^{i}} N_{L}(x, p)+\frac{1}{16 p_{0}^{2}} \frac{\partial}{\partial p^{i}} \frac{\partial}{\partial p^{i}} \tau^{i i} N_{L}(x, p)=0 .
\end{gathered}
$$

Here for convenience we divided the r.h.s. of Eq. (33) on $p_{0}$. Just to demonstrate that the solution of the above equation can be associated with the isotropic steady state, let us find an approximate analytic solution of Eq. (33) for $|\mathbf{p}| /\left|p_{0}\right| \ll 1$. One can easily see that it is

$$
N_{L}(x, p) \sim \exp \left[-2 p_{0}\left(\frac{p_{0} \partial^{0} \varrho(x)}{\tau^{00}}+\frac{2 p_{i} \partial^{i} \varrho(x)}{\tau^{i i}}\right)\right] .
$$

Notice, that such a steady state is obtained without account of energy-momentum dispersion relation. So, it is valid, in fact, only if mass shell constraint on $p_{\mu}$ is not strongly peaked like the delta-function but, instead, is wide enough, having, however, some limited virtuality.

For an expanding system one can expect that $\partial^{\mu} \varrho>0$ because $\varrho<0$, see (16) and (18). Then, this steady state has quasi - local equilibrium form with "effective temperature" that is $\sim 1 / p_{0}$, and "effective collective four-velocity" that is $\sim \partial^{\mu} \varrho$, and can be related to 
the so-called prethermalization stage [37]. Also, one can see that (35) demonstrates spatiotemporal decoherence of the long-wavelength subsystem. Indeed, the lengths of coherence are associated with values of the off-diagonal elements of the corresponding density matrix, $\rho_{L}\left(x+\frac{1}{2} \Delta x, x-\frac{1}{2} \Delta x\right)=\int d^{4} p \exp (i p \Delta x) N_{L}(x, p)$. The structure like (35) for $N_{L}(x, p)$ leads typically to finite coherence lengths. Because we do not fix the dispersion relation, we just illustrate our conclusion analytically supposing particles on a zero-mass shell. Then, calculating the density matrix in the "rest frame", it is easy to see that in the time direction nondiagonal elements of the density matrix will be proportional to $e^{-\Delta t^{2} / \lambda_{t}^{2}}$ with temporal correlation length in this rest frame system to be $\lambda_{t} \propto \sqrt{\partial^{0} \rho / \tau^{00}}$. During this time the long-length state loses the coherence. In space directions there are also exponential cuts in nondiagonal elements of the density matrix. So one can conclude that coherence lengths in this steady state are finite and are caused by the hydrodynamic-like parameters and energymomentum dispersion relation. In a similar way, thermal wave length $\lambda_{t h} \propto 1 / \sqrt{m T}$ defines the off-diagonal elements of the corresponding density matrix and, so, the spatial coherence lengths of the non relativistic Boltzmann distribution.

One can see that (35) is the isotropic expression in the locally co-moving fluid-like rest frame. It is reasonable to expect that for such a steady state the energy momentum tensor (14) develops a sufficient degree of isotropy in the locally co-moving frame. Because approximate isotropy in the locally co-moving frame and decoherence of densities are the basic premises for applicability of hydrodynamics, one can conjuncture that the energy momentum tensor of the long-wavelength modes can, eventually, approach to the energy momentum tensor of an effective viscous fluid.

\section{CONCLUSIONS}

In this paper, we discuss the physical mechanism that can explain the source of decoherence at the early stage of matter evolution in relativistic nucleus-nucleus collisions, and the subsequent approach to hydrodynamical behavior. Our method, while admittedly heuristic, provides a physical understanding of the decoherence phenomenon, which was lacking in the current attempts of description of thermalization in $A+A$ collisions, and sheds some light on the mechanism of isotropization. In our opinion, understanding of the dynamical mechanisms of decoherence and thermalization should create the necessary prerequisites for 
unambiguous calculation of viscous coefficients in $A+A$ collisions, and we hope that our analysis can be useful for this aim.

Let us sum up our main points. First, it is well known that decoherence of local densities is a necessary condition for thermalization, and in an isolated system that is governed as whole by the unitary quantum dynamics, the only possibility for decoherence is decomposition of the system into subsystems. An ambiguity of a splitting procedure is removed by the requirement that such a splitting must be done in an observer dependent way. Taking into account typical observational conditions, we proposed to split the system created in a relativistic $A+A$ collision into a long-length modes subsystem and a short-length modes subsystem, and consider the former as a relevant subsystem. Because the long-length modes in the initial stage of a relativistic $A+A$ collision are highly populated [5], this allows us to consider evolution of the corresponding expectation value of the quantum field in the quasiclassical approximation with noise term. The latter is associated with quantum fluctuations that are mostly contributed by the irrelevant (from an observational viewpoint) short-length modes 5 We suppose that such a stochasticity accounts effectively for quantum entanglement between different scales. Then, entanglement-driven stochasticity results in irreversibility and decoherence for the effective coarse-grained dynamics of the large scales.

We demonstrate how such a physical mechanism works by means of scalar field model 6 We derived an evolution equation of the Fokker-Planck type for the Wigner function of the relevant part of the system and demonstrated, after some simplifying assumptions, that this equation can describe decoherence and isotropization at prethermalization stage, which are necessary conditions for eventual thermalization and hydrodynamics. Notice that this happens as result of interactions with the irrelevant (i.e., observationally inaccessible) degrees of freedom, and no averaging over the ensemble of initial conditions is needed for such a quantum thermalization. The generated non-zero entropy can be understood as the entanglement entropy of the long-length subsystem of the system created by a nucleusnucleus collision, while the entropy of the whole closed system does not change with time due to the unitarity of the time evolution.

Our analysis supports the idea that thermalization and transition to hydrodynamics are contextual, and are related to a particular procedure of decomposition of the whole quantum ${ }^{5}$ See also Ref. [38] where it was proposed that unobservation of higher-order correlators may result in effective decoherence and associated entropy production in quantum field theory.

6 One can adjust this analysis for QCD systems utilizing quark-gluon Wigner functions, see Ref. [39]. 
system into subsystems that contain a large enough number degrees of freedom (evidently, one should not expect a similar behavior in systems with few degrees of freedom). Because observables are measured with some degree of precision (and not all possible observables are measured) in typical experiment, this leaves the room for inaccessible degrees of freedom, and, so, allows for hydrodynamical approximation. A fluid dynamics then appears as an effective long-wavelength theory. One can expect that the utilization of a full unitary quantum evolution of a closed system with subsequent projection into relevant coarsegrained subspace at the measurement will result in the same predictions for a statistical ensemble of experimental data as utilization of a relevant coarse-grained effective theory that follows to instantaneous decomposition of the whole state into relevant and irrelevant subsystems/observables (for more discussions see Ref. [40]). On the other hand, a hydrodynamical description is inappropriate for an observer who wholly measures the total set of observables for an isolated quantum system. Such an observer then will have to calculate the whole quantum evolution of a system of interest to predict results of such a "complete" experiment.

\section{ACKNOWLEDGMENTS}

Yu.S. thanks J. Maldacena, A. Ashtekar, P. Braun-Munzinger for useful discussions of the paper subject, and ExtreMe Matter Institute EMMI for the visiting professor position. The research was carried out within the scope of the EUREA: European Ultra Relativistic Energies Agreement (European Research Group: "Heavy Ions at Ultrarelativistic Energies") and is supported by the National Academy of Sciences of Ukraine (Agreement - 2014).

[1] U. Heinz, in "Relativistic Heavy Ion Physics", Landolt-Boernstein New Series, Vol. I/23, edited by R. Stock (Springer Verlag, New York, 2010), Chap. 5 arXiv:0901.4355; D.A. Teaney, arXiv:0905.2433; H. Song, arXiv:1207.2396, U. Heinz, R. Snellings, Annu. Rev. Nucl. Part. Sci. 63 (2013) 123 arXiv:1301.2826]; C. Gale, S. Jeon, B. Schenke, Int. J. Mod. Phys. A 28 (2013) 1340011 arXiv:1301.5893.

[2] B. Müller, A. Schäfer, Int. J. Mod. Phys. E 20 (2011) 2235 arXiv:1110.2378]; J. Berges, J.-P. Blaizot, F. Gelis, J. Phys. G: Nucl. Part. Phys. 39 (2012) 085115 [arXiv:1203.2042]. 
[3] A. El, Z. Xu, C. Greiner, Nucl. Phys. A 806 (2008) 287 arXiv:0712.3734]; Z. Xu, L. Cheng, A. El, K. Gallmeister, C. Greiner, J. Phys. G 36 (2009) 064035 arXiv:0812.3839.

[4] St. Mrówczyński, Acta Phys. Polon. B 37 (2006) 427 arXiv:hep-ph/0511052; S.V. Akkelin, Phys. Rev. C 78 (2008) 014906 arXiv:0801.1628]; St. Mrówczyński, B. Müller, Phys. Rev. D 81 (2010) 065021 [arXiv:0912.5108]; J. Peralta-Ramos, E. Calzetta, Phys. Rev. D 86 (2012) 125024 arXiv:1208.2715] M. Attems, A. Rebhan, M. Strickland, Acta Phys. Polon. Supp. 6 (2013) 393 [arXiv:1302.5098].

[5] F. Gelis, E. Iancu, J. Jalilian-Marian, R. Venugopalan, Ann. Rev. Nucl. Part. Sci. 60 (2010) 463 arXiv:1002.0333]; T. Lappi, Int. J. Mod. Phys. E 20 (2011) 1 arXiv:1003.1852] ; L. McLerran, Prog. Theor. Phys. Suppl. 187 (2011) 17 arXiv:1011.3204]; F. Gelis, Int. J. Mod. Phys. A 28 (2013) 1330001 [arXiv:1211.3327].

[6] J.R. Klauder, E.C.G. Sudarshan, Fundamentals of Quantum Optics (Benjamin New York, 1968).

[7] K. Hepp, Commun. Math. Phys. 35 (1974) 265; L.G. Yaffe, Rev. Mod. Phys. 54 (1982) 407.

[8] F. Gelis, arXiv:1312.5497; J. Berges, K. Boguslavski, S. Schlichting, R. Venugopalan, arXiv:1311.3005; arXiv:1312.5216.

[9] K. Gottfried, T.-M. Yan, Quantum Mechanics: Fundamentals (Springer-Verlag New York, 2003).

[10] W.H Zurek, J.P. Paz, Phys. Rev. Lett. 72 (1994) 2508 arXiv:gr-qc/9402006; F. Cooper, J. Dawson, S. Habib, R.D. Ryne, Phys. Rev. E 57 (1998) 1489 arXiv:quant-ph/9610013].

[11] S. Habib, K. Shizume, W.H. Zurek, Phys. Rev. Lett. 80 (1998) 4361 arXiv:quant-ph/9803042].

[12] J.P. Paz, W.H. Zurek, arXiv:quant-ph/0010011.

[13] J.M. Maldacena, Adv. Theor. Math. Phys. 2 (1998) 231 arxiv:hep-th/9711200; E. Witten, Adv. Theor. Math. Phys. 2 (1998) 253 arXiv:hep-th/9802150.

[14] O. Aharony, S.S. Gubser, J. Maldacena, H. Ooguri, Y. Oz, Phys. Rep. 323 (2000) 183 arXiv:hep-th/9905111]; J.M. Maldacena, arXiv:hep-th/0309246; arXiv:1106.6073.

[15] J. Casalderrey-Solana, H. Liu, D. Mateos, K. Rajagopal and U. A. Wiedemann, arXiv:1101.0618; A. Bernamonti, R. Peschanski, Nucl. Phys. Proc. Suppl. 216 (2011) 94 arXiv:1102.0725]; O. DeWolfe, S.S. Gubser, C. Rosen, D. Teaney, arXiv:1304.7794.

[16] J.-P. Blaizot, Acta Physica Polonica B Proceedings Supplement 4 (2011) 641 arXiv:1108.3049. 
[17] V. Balasubramanian, P. Kraus, Phys. Rev. Lett. 83 (1999) 3605 arXiv:hep-th/9903190; T. Faulkner, H. Liu, M. Rangamani, JHEP 1108 (2011) 051 arXiv:1010.4036]; I. Heemskerk, J. Polchinski, JHEP 1106 (2011) 031 arXiv:1010.1264]; L. Susskind, E. Witten, arXiv:hep-th/9805114; I. Papadimitriou, K. Skenderis, arXiv:hep-th/0404176.

[18] S. Bhattacharyya, V.E. Hubeny, S. Minwalla, M. Rangamani, JHEP 0802 (2008) 045 arXiv:0712.2456]; M. Rangamani, Class. Quant. Grav. 26 (2009) 224003 arXiv:0905.4352]; V.E. Hubeny, M. Rangamani, Adv. High Energy Phys. 2010 (2010) 297916 [arXiv:1006.3675; V.E. Hubeny, S. Minwalla, M. Rangamani, arXiv:1107.5780; V.E. Hubeny, Class. Quant. Grav. 28 (2011) 114007 arXiv:1011.4948.

[19] W.G. Unruh, Phys. Rev. D 14 (1976) 870.

[20] L.C.B. Crispino, A. Higuchi, G.E.A. Matsas, Rev. Mod. Phys. 80 (2008) 787 arXiv:0710.5373].

[21] W.G. Unruh, R.M. Wald, Phys. Rev. D 29 (1984) 1047.

[22] Yu.M. Sinyukov, J. Phys. A: Math. Gen. 15 (1982) 2533.

[23] D. Kharzeev, K. Tuchin, Nucl. Phys. A 753 (2005) 316 arXiv:hep-ph/0501234; D. Kharzeev, Nucl. Phys. A 774 (2006) 315 arXiv:hep-ph/0511354.

[24] S. Ryu, T. Takayanagi, Phys. Rev. Lett. 96 (2006) 181602 arXiv:hep-th/0603001; JHEP 0608 (2006) 045 arXiv:hep-th/0605073.

[25] T. Takayanagi and T. Ugajin, JHEP 1011 (2010) 054 arXiv:1008.3439].

[26] T. Nishioka, S. Ryu, T. Takayanagi, J. Phys. A 42 (2009) 504008 arXiv:0905.0932] T. Takayanagi, Class. Quant. Grav. 29 (2012) 153001 arXiv:1204.2450].

[27] S. Bhattacharyya, R. Loganayagam, I. Mandal, S. Minwalla, A. Sharma, JHEP 0812 (2008)116 arXiv:0809.4272] R. Baier, P. Romatschke, D. T. Son, A. O. Starinets, M. A. Stephanov, JHEP 0804 (2008) 100 [arXiv:0712.2451]; I. Bredberg, C. Keeler, V. Lysov, A. Strominger, JHEP 1103 (2011) 141 [arXiv:1006.1902]; P. Kovtun, J. Phys. A: Math. Theor. 45 (2012) 473001 arXiv:1205.5040.

[28] E. Joos, H.D. Zeh, Z. Phys. B 59 (1985) 223; W.H. Zurek, Rev. Mod. Phys. 75 (2003) 715 arXiv:quant-ph/0105127; D. Guilini, E. Joos, C. Kiefer, J. Kupsch, I.-O. Stamatescu, H.D. Zeh, Decoherence and the Appearance of a Classical World in Quantum Theory (Springer-Verlag Berlin Heidelberg, 1996); M. Schlosshauer, Rev. Mod. Phys. 76 (2004) 1267 arXiv:quant-ph/0312059]; H.D. Zeh, The Physical Basis of the Direction of Time(SpringerVerlag Berlin Heidelberg, 2007). 
[29] B. d'Espagnat, Phys. Rep. 110 (1984) 201; Conceptual Foudations of Quantum Mechanics (Perseus Books, 1999).

[30] J. Gemmer, M. Michel, G. Mahler, Quantum Thermodynamics. Emergence of Thermodynamic Behavior Within Composite Quantum Systems. Lect. Notes Phys. 657 (Springer Berlin Heidelberg, 2004).

[31] F. Lombardo, F. Mazzitelli, Phys. Rev. D 53 (1996) 2001 arXiv:hep-th/9508052; E.A. Calzetta, B.L. Hu, F.D. Mazzitelli, Phys. Rep. 352 (2001) 459 arXiv:hep-th/0102199]; E. Calzetta and B.-L. Hu, Nonequilibrium Quantum Field Theory (Cambridge University Press, 2008).

[32] H.-Th. Elze, arXiv:quant-ph/9710063.

[33] H. Grabert, Projection Operator Techniques in Nonequilibrium Statistical Mechanics (Springer Berlin Heidelberg, 1982); J. Rau, B. Müller, Phys. Rep. 272 (1996) 1 [arXiv:nucl-th/9505009].

[34] A. Vikman, Europhys. Lett. 101 (2013) 34001 arXiv:1208.3647].

[35] R. Balian, Y. Alhassid, H. Reinhardt, Phys. Rep. 131 (1986) 1.

[36] S.R. de Groot, W.A. van Leeuwen, Ch. G. van Weert, Relativistic Kinetic Theory (NorthHolland Publ. Comp. Amsterdam, 1980); H.-Th. Elze, AIP Conf. Proc. 631 (2002) 229 arXiv:hep-ph/0204309.

[37] J. Berges, S. Borsanyi, C. Wetterich, Phys. Rev. Lett. 93 (2004) 142002 hep-ph/0403234.

[38] A. Giraud, J. Serreau, Phys. Rev. Lett. 104 (2010) 230405 arXiv:0910.2570].

[39] H.-Th. Elze, M. Gyulassy, D. Vasak, Phys. Lett. B 177 (1986) 402; H.-Th. Elze, Z. Phys. C 38 (1988) 211; H.-Th. Elze, U. Heinz, Phys. Rep. 183 (1989) 81; H.-Th. Elze, Z. Phys. C 47 (1990) 647; S. Ochs, U. Heinz, Annals Phys. 266 (1998) 351 arXiv:hep-th/9806118.

[40] J.P. Paz, W.H. Zurek, Phys. Rev. D 48 (1993) 2728 arXiv:gr-qc/9304031. 